Cite this: Phys. Chem. Chem. Phys., 2014, 16, 5627

Received 28th November 2013 Accepted 27th January 2014

DOI: $10.1039 / c 3 c p 55018 d$

www.rsc.org/pccp

\title{
Solvent-induced conformational changes in cyclic peptides: a vibrational circular dichroism study $\dagger$
}

\author{
Christian Merten, ${ }^{a b}$ Fee Li, ${ }^{\text {b }}$ Kenny Bravo-Rodriguez, ${ }^{c}$ Elsa Sanchez-Garcia, ${ }^{\circ}$ \\ Yunjie $\mathrm{Xu}^{* a}$ and Wolfram Sander ${ }^{\mathrm{b}}$
}

\begin{abstract}
The three-dimensional structure of a peptide is strongly influenced by its solvent environment. In the present study, we study three cyclic tetrapeptides which serve as model peptides for $\beta$-turns. They are of the general structure cyclo(Boc-Cys-Pro-X-Cys-OMe) with the amino acid X being either glycine (1), or L- or D-leucine (L- or D-2). Using vibrational circular dichroism (VCD) spectroscopy, we confirm previous NMR results which showed that D-2 adopts predominantly a $\beta_{\|}$turn structure in apolar and

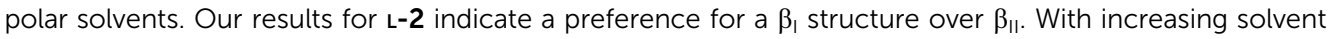
polarity, the preference for 1 is shifted from $\beta_{\|}$towards $\beta_{l}$. This conformational change goes along with the breaking of an intramolecular hydrogen bond which stabilizes the $\beta_{\| I}$ conformation. Instead, a hydrogen bond with a solvent molecule can stabilize the $\beta_{\text {I }}$ turn conformation.
\end{abstract}

\section{Introduction}

Besides periodic secondary structures like $\alpha$-helices and $\beta$-sheets, turns are the most common structural motifs in peptides and as such they establish the directional reversal in peptide strands. Most attention in studies on turns has been paid to the tight $\beta$-turns which link the strands of antiparallel $\beta$-sheet structures. They are also exposed to the environment of peptides, i.e. the solvent, ions, and substrates. $\beta$-Turns consist of four amino acid residues linked by three amide bonds and show a high structural flexibility. Initially, $\beta$-turns were assumed to form a hydrogen bond between the carbonyl group of the first (position $i$ ) and the amide $\mathrm{N}-\mathrm{H}$ of the fourth amino acid residue (position $i+3) .{ }^{1}$ However, it was later shown that $\beta$-turns without this hydrogen bond exist as well. ${ }^{2}$ Nowadays, eight different tight $\beta$-turns are distinguished and characterized mainly by the dihedral angles adopted by the amino acid residues $i+1$ and $i+2$. $^{3}$ The amino acid sequences and its peptidic and non-peptidic environments determine which $\beta$-turn conformation is adopted under certain conditions.

The most common experimental techniques used to study peptide conformations are infrared and Raman spectroscopy, ${ }^{3}$ electronic circular dichroism (ECD) and two-dimensional NMR spectroscopy. The chiroptical versions of infrared and Raman

\footnotetext{
${ }^{a}$ Department of Chemistry, University of Alberta, Edmonton, Alberta, T6G2G2, Canada.E-mail: yunjie.xu@ualberta.ca

${ }^{b}$ Ruhr-Universität Bochum, Fakultät für Chemie und Biochemie, 44801 Bochum, Germany. E-mail: christian.merten@ruhr-uni-bochum.de

${ }^{c}$ Max-Planck-Institut für Kohlenforschung, 45470 Mülheim an der Ruhr, Germany $\dagger$ Electronic supplementary information (ESI) available: Additional comparative plots for experimental spectra, Raman spectra of $\mathbf{1}$ and D-2, calculated VCD spectra. See DOI: 10.1039/c3cp55018d
}

spectroscopy, vibrational circular dichroism (VCD) spectroscopy and Raman optical activity (ROA), which measure vibrational optical activity (VOA), ${ }^{4}$ have also become well-known and powerful techniques to study peptides, proteins ${ }^{5-8}$ and carbohydrates ${ }^{9}$ as well as other chiral molecules in solution..$^{10-17}$

Small peptides can serve as structural model systems for examining the influence of the peptide environment on the conformations of turns. They can easily be synthesized and studied in much greater detail using spectroscopic and theoretical methods. In many cases, small peptides have also been used to establish structure-spectra relationships. For instance, the CD spectra of pure $\beta_{\mathrm{I}}$ and $\beta_{\mathrm{II}}$ turns have been determined by Perczel et al. in their studies on cyclic hexapeptides ${ }^{18}$ confirming the theoretical predictions by Woody. ${ }^{19,20}$ Diem et al. used cyclic tri-, tetra-, and pentapeptides to establish the VCD spectral pattern of the amide I vibrations of $\beta$-turns. ${ }^{21-23}$

Recently, we introduced the model peptide cyclo(Boc-CysPro-Gly-Cys-OMe) 1 (Fig. 1), ${ }^{24}$ a cyclic disulfide-bridged tetrapeptide of which the two derivatives cyclo(Cys-Pro-Gly-Cys) ${ }^{23}$ (1a) and cyclo(Boc-Cys-Pro-Gly-Cys-NHMe $)^{25}(\mathbf{1 b})$ have been studied before. For the unprotected form 1a of the tetrapeptide, Diem et al. assumed a mixture of $\beta_{\mathrm{I}}$ and $\beta_{\mathrm{II}}$ turn structures in DMSO. This assignment was based on a comparison of the amide I VCD pattern with the ones measured for cyclo(Cys-Pro-Phe-Cys) and cyclo(CysPro-D-Phe-Cys) in DMSO and $\mathrm{DMSO} / \mathrm{CDBr}_{3}$, which contain mainly either $\beta_{\mathrm{I}}$ or $\beta_{\mathrm{II}}$ turn structures, respectively. The cyclo(Boc-Cys-ProGly-Cys-NHMe) derivative $\mathbf{1 b}$, on the other hand, was assigned to a $\beta_{\text {I }}$ structure based on the lack of experimental evidence for nuclear Overhauser effects (NOE) between the Pro- ${ }^{\alpha} \mathrm{H}$ and Gly-NH.

In contrast to these previously studied derivatives, $\mathbf{1}$ is highly soluble in non-polar chloroform as well as in polar solvents 


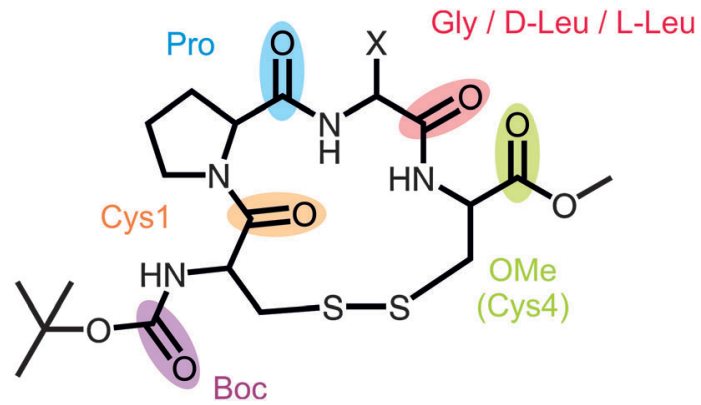

Fig. 1 General structure of the investigated cyclic tetrapeptides with $\mathrm{X}=\mathrm{H}$ for $\mathbf{1}$ and $\mathrm{X}=$ iso-butyl for $\mathrm{D}-$ and $\mathrm{L}-\mathbf{2}$.

such as acetonitrile and DMSO. This allowed us to investigate the effect of solvents and temperature on the turn conformation with different techniques such as 2D-NMR, IR, and CD spectroscopy, as well as Car-Parrinello molecular dynamics. ${ }^{24}$ It has been shown that 1 adopts a $\beta_{\text {II }}$ structure in solution and in the solid state and that a hydrogen bond between Cys1- $\mathrm{C}=\mathrm{O}$ and Cys4-NH stabilizes the turn conformation of the cyclic peptide. While Cys1- $\mathrm{C}=\mathrm{O}$ forms another hydrogen bond with the $\mathrm{NH}$-bond of Gly of the neighboring molecule in solid state, in solution, however, the carbonyl group competes with solvent molecules for the hydrogen bonding donor.

The present work complements this initial investigation by applying VCD spectroscopy to investigate the preferred conformations of the cyclic peptide 1 in solvents of different polarities, i.e. chloroform, acetonitrile and DMSO. Additionally, two related peptides ( $\mathbf{L}-2$ and $\mathbf{D}-2$ ) with $\mathrm{L}^{-}$or D-leucine instead of glycine are studied here. Unlike NMR and ECD, VCD spectroscopy can be used to compare the peptides in all three solvents. For instance, the peaks of some $\mathrm{NH}$ protons could not be resolved in the reported NMR spectra of $\mathbf{1}$ in chloroform. The UV and ECD bands of the peptides were also masked by bands from chloroform or DMSO.

In this paper, the VCD analysis of the three tetrapeptides is carried out first without making any assumptions based on the previous studies (except the crystal structure of 1). Afterwards, the obtained results are compared to the NMR experiments and molecular dynamics simulations. Our VCD analysis thus provides new evidence to establish more conclusively the $\beta$-turn structures of $\mathbf{1}$ and $\mathbf{2}$ in acetonitrile and the role of the solvent on the conformation of the peptides.

\section{Experimental and computational details}

The synthesis procedures of $\mathbf{1}, \mathbf{L}-\mathbf{2}$, and $\mathbf{D}-\mathbf{2}$ were reported elsewhere. ${ }^{24,26}$ Deuterated solvents were purchased from SigmaAldrich and Cambridge Isotopes.

The IR and VCD spectra of $\mathbf{1}, \mathbf{L}-\mathbf{2}$, and $\mathbf{D}-\mathbf{2}$ were measured at a resolution of $4 \mathrm{~cm}^{-1}$ in the fingerprint region (1800-1100 $\mathrm{cm}^{-1}$ ) using a Bruker Vertex 70 FTIR spectrometer equipped with a PMA50 module for polarization modulated measurements. The photoelastic modulator was set to an optimum working frequency of $1500 \mathrm{~cm}^{-1}$. The spectra were recorded in the solvents $\mathrm{CDCl}_{3}$, $\mathrm{CD}_{3} \mathrm{CN}$, and DMSO- $\mathrm{d}_{6}$ under otherwise identical conditions, i.e. a concentration of $45 \mathrm{mg} \mathrm{ml}^{-1}(\sim 0.1 \mathrm{mM})$, a path length of $100 \mu \mathrm{m}$, and an accumulation time of 4 hours ( $\sim 25 \mathrm{k}$ scans). Background-correction by subtracting solvent spectra has been carried out for the IR spectra while the VCD spectra are shown as obtained.

Geometry optimizations and spectra calculations were performed at the B3LYP/6-311++G(2d,p) level of theory using the Gaussian 09 (Rev. C.01) software package. ${ }^{27}$ Dispersion-correction has been taken into account at the DFT-D level using the $\operatorname{iop}(3 / 124=3)$ setting; however, the correction did not significantly affect the calculated VCD pattern in the amide I and amide II regions. Implicit solvation effects have been accounted for by using the polarizable continuum model (IEFPCM) for chloroform and acetonitrile. ${ }^{28-30}$ The normal mode frequencies obtained were scaled by a factor of 0.98 for a better visual comparison with the experimental spectra. Line broadening has been taken into account by assigning a Lorentzian band shape with a half-width at half-height of $8 \mathrm{~cm}^{-1}$ to the calculated dipole and rotational strengths.

The dynamical behaviour of $\mathbf{1}$ in acetonitrile was investigated by Replica Exchange Molecular Dynamics (REMD) simulations carried out for $60 \mathrm{~ns}$ using the Gromacs4.6 $\operatorname{code}^{31-33}$ and the OPLSA force field. ${ }^{34}$ The temperature range was $290-400 \mathrm{~K}$ and the temperature distribution was established according to the Patriksson and van der Spoel approach. ${ }^{35}$ Although originally intended for water, this approach allowed for a good exchange ratio between replicas in acetonitrile too (see the ESI $\dagger$ for replica exchange statistics). The reported crystal structure of $\mathbf{1}^{24}$ was used as the starting point for the simulations. For the treatment of electrostatic and van der Waals interactions the PME method ${ }^{36}$ and a twin range cut-off ${ }^{37}$ were used, respectively, with cut-off distances of $1.2 \mathrm{~nm}$ in both cases. The exchange of replicas was attempted every 1 ps. The time step for all simulations was 2 fs. Bonds containing hydrogen atoms were kept fixed using the LINCS algorithm. ${ }^{38}$ The cluster analysis of the results was performed using the Gromos method. ${ }^{39}$

\section{Results and discussion}

\section{Experimental IR and VCD spectra}

The fingerprint region of the infrared spectrum of a peptide can be divided into the carbonyl/amide I region (1800-1600 $\mathrm{cm}^{-1}$ ), the amide II region (1600-1480 $\mathrm{cm}^{-1}$ ) which is characterized by $\mathrm{N}-\mathrm{H}$ bending and $\mathrm{C}-\mathrm{N}$ stretching vibrations, and the region below $1480 \mathrm{~cm}^{-1}$ which contains the amide III region $\left(1350-1250 \mathrm{~cm}^{-1}\right)$ and arises from complex peptide backbone as well as side group vibrations. For the present study, we focus on the amide I and amide II regions since they are very characteristic for the hydrogen bonding network of the peptide, and comment only briefly on the region below $1480 \mathrm{~cm}^{-1}$.

Fig. 2 compares the experimental IR and VCD spectra of $\mathbf{1}, \mathbf{L}-\mathbf{2}$, and $\mathrm{D}-2$ measured in the three different solvents $\mathrm{CDCl}_{3}, \mathrm{CD}_{3} \mathrm{CN}$, and DMSO- $\mathrm{d}_{6}$. Direct comparisons for the IR spectra of 1 and D-2 are provided in the supporting information (Fig. S1, ESI $\dagger$ ). 

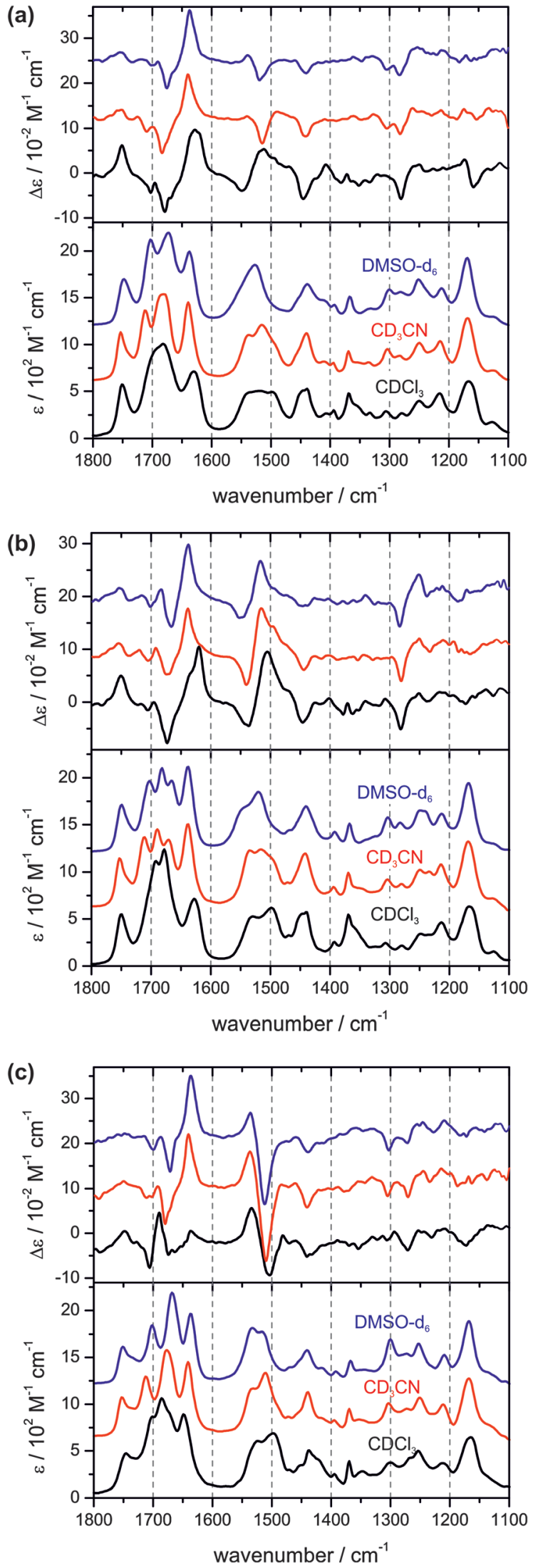

Fig. 2 Experimental VA and VCD spectra of $\mathbf{1}$ (top), D-2 (middle), and L-2 (bottom) in $\mathrm{CDCl}_{3}, \mathrm{CD}_{3} \mathrm{CN}$, and DMSO- $\mathrm{d}_{6}$.
Table 1 Vibrational band positions for 1, D-, and L-2 in the amide I and II regions in $\mathrm{CDCl}_{3}(\mathrm{~A}), \mathrm{CD}_{3} \mathrm{CN}(\mathrm{B})$, and $\mathrm{DMSO}-\mathrm{d}_{6}(\mathrm{C})$. Wavenumbers are given in $\mathrm{cm}^{-1}$

\begin{tabular}{|c|c|c|c|c|c|c|c|c|c|}
\hline & \multicolumn{3}{|c|}{1 (X = Gly) } & \multicolumn{3}{|c|}{ D-2 (X = D-Leu $)$} & \multicolumn{3}{|c|}{ L-2 (X = L-Leu) } \\
\hline & A & B & $\mathrm{C}$ & A & B & $\mathrm{C}$ & A & B & $\mathrm{C}$ \\
\hline Ester $\mathrm{C}=\mathrm{O}$ & 1751 & 1753 & 1747 & 1749 & 1753 & 1749 & 1745 & 1753 & 1751 \\
\hline Boc $\mathrm{C}=\mathrm{O}$ & 1703 & 1711 & 1703 & 1706 & 1711 & 1703 & 1703 & 1713 & 1701 \\
\hline Amide I X & & & & 1691 & 1689 & 1682 & 1686 & & \\
\hline Amide I Pro & 1682 & 1680 & 1672 & 1678 & 1670 & 1666 & 1672 & 1676 & 1668 \\
\hline Amide I Cys1 & 1630 & 1639 & 1637 & 1628 & 1637 & 1637 & 1649 & 1641 & 1637 \\
\hline \multirow[t]{3}{*}{ Amide II } & 1548 & 1537 & 1539 & 1529 & 1535 & 1544 & 1524 & 1534 & 1534 \\
\hline & 1512 & 1515 & 1520 & 1505 & 1516 & 1520 & & 1514 & 1519 \\
\hline & 1496 & 1491 & & 1498 & 1494 & & 1496 & & \\
\hline
\end{tabular}

For the spectra of $\mathbf{1}$, it has been ensured that the spectral pattern did not show any concentration dependence. For all three peptides, distinct changes in the amide I and II regions of the IR spectra can be observed when the solvent is changed from non-polar chloroform to either of the polar solvents. The band assignments discussed below are summarized in Table 1 .

First, we examine the amide I regions of the IR spectra of the peptides and the solvent-induced changes. The carbonyl vibration of the methyl ester group of Cys(4) appears to be unaffected by the different solvents and is found to be at $1750 \mathrm{~cm}^{-1}$ in all spectra. The carbonyl vibration of Cys(1) observed at $1627 \mathrm{~cm}^{-1}$ in chloroform- $\mathrm{d}_{1}$ is blue-shifted by about $10 \mathrm{~cm}^{-1}$ in the polar solvents and is slightly narrower. The most prominent changes occur in the region 1750-1650 $\mathrm{cm}^{-1}$. In the spectrum of 1 in chloroform $-\mathrm{d}_{1}$, this region is dominated by a broad band with an intensity maximum at $1680 \mathrm{~cm}^{-1}$ and shoulders at the higher wavenumber side. In acetonitrile- $\mathrm{d}_{3}$, a sharp feature at $1712 \mathrm{~cm}^{-1}$ separates from the broad band which is still found to be centred at $1680 \mathrm{~cm}^{-1}$ (1703 and $1672 \mathrm{~cm}^{-1}$ in DMSO-d $\mathrm{d}_{6}$. The amide I region of $\mathrm{L}-2$ behaves quite similarly. For peptide $\mathbf{D}-2$, an intense band with two maxima at 1678 and $1691 \mathrm{~cm}^{-1}$ and a shoulder at the higher wavenumber side is observed in chloroform which splits into three bands found at 1670,1689 , and $1711 \mathrm{~cm}^{-1}$ in the spectra measured in acetonitrile- $\mathrm{d}_{3}\left(1666,1682\right.$, and $1703 \mathrm{~cm}^{-1}$ in DMSO- $\mathrm{d}_{6}$ ). From higher to lower wavenumbers, these three bands are assigned to the carbonyl vibration of the Boc-group, Pro, and the amino acid Gly respectively $\mathrm{D} / \mathrm{L}$-Leu.

Second, we consider the VCD spectra of the amide I region of the three peptides. The experimental spectra taken in chloroform feature a strong positive band for the carbonyl vibration of the methyl ester group of Cys(4) which becomes weaker in polar solvents. For the Cys(1) carbonyl vibration, a strong positive VCD is observed for $\mathbf{1}$ and $\mathbf{D}-\mathbf{2}$ which shows as two closely spaced positive bands in chloroform. For $\mathrm{L}-2$, the intensity of the VCD of Cys(1) in chloroform is much weaker and becomes stronger in polar solvents. The VCD pattern in the $1750-1650 \mathrm{~cm}^{-1}$ region is rather complex. The VCD band assigned to the carbonyl vibration of the variable amino acid residue, Gly or Leu, is in all cases negative. However, for 1 the negative band at $1678 \mathrm{~cm}^{-1}$ features a small negative shoulder at $1669 \mathrm{~cm}^{-1}$. For the carbonyl vibration of the Boc-group, a weak negative VCD is observed, while the VCD band of the carbonyl vibration of Pro 
can only be clearly identified as the positive band in the spectra of $\mathbf{L}-2$.

For the amide I region, it can be concluded that the IR and VCD spectra do not show clear evidence for significant conformational changes due to solute-solvent interactions. Furthermore, a direct comparison with the amide I VCD spectra reported by Diem et al. for the unprotected version 1a is difficult because of the unknown influence of the Boc-group on the amide I vibrations.

Therefore, we now turn to the amide II region which features three vibrational modes assigned to the Cys(4), the variable amino acid residue Gly respectively D/L-Leu, and Cys(1). In all sets of IR spectra, the amide II vibrations are found to blend into one very broad band. However, a clear blue-shift of the bands can be observed when going from $\mathrm{CDCl}_{3}$ over $\mathrm{CD}_{3} \mathrm{CN}$ towards DMSO- $\mathrm{d}_{6}$. The corresponding VCD spectra of the amide II vibrations show very significant changes upon substitution of the solvent. The region features a dominant $-/+/+$ pattern in all spectra of peptide $\mathbf{D}-2$, and a $+/-$ pattern for $\mathbf{L}-2$. The spectral pattern of peptide 1 , however, clearly changes from $-/+/+$ in chloroform- $\mathrm{d}_{1}$ to $+/-/+$ in the two polar solvents. This observation is a strong indicator for a solvent-induced conformational change of the tetrapeptide's cyclic backbone which is only possible for $\mathbf{1}$ but not for $\mathbf{D}-$ or $\mathbf{L}-\mathbf{2}$.

Below $1480 \mathrm{~cm}^{-1}$, no significant changes can be observed in the IR spectra. In light of the above mentioned spectral changes of $\mathbf{1}$, it is noteworthy that the amide III region of $\mathbf{1}$ is similar to that of $\mathrm{L}-2$. The VCD pattern found that the $1385-1330 \mathrm{~cm}^{-1}$ region of the chloroform measurements of 1 and 2 compared to the lack of VCD bands in polar solvents could carry further interesting structural information which, however, we will not address any further in this study.

Our experimental spectra thus suggest that the conformational equilibria in $\mathbf{D}-$ and $\mathbf{L}-\mathbf{2}$ are much less sensitive to solvent-induced conformational changes than in $\mathbf{1}$. By contrast, we can clearly establish that the conformational preferences of $\mathbf{1}$ very much depend on the solvent environment.

\section{Conformational analysis}

In order to further interpret the experimental VCD spectra, density functional theory (DFT) based spectra calculations were carried out. ${ }^{40-42}$ Since VCD spectroscopy is very sensitive to conformational changes, a thorough conformational analysis of 1 and subsequent spectra calculations were performed.

The starting point for the analysis was the crystal structure of $1,{ }^{24}$ which has been optimized at the B3LYP-D/6-311++G(2d,p) level of theory in the gas phase. The obtained optimized geometry, denoted $\mathrm{r} 1-\mathrm{c} 1$, is shown in Fig. 3. As the comparison of the $\phi$ and $\psi$ angles in Table 2 shows, a $\beta_{\text {II }}$ turn structure is adopted in the solid state and in the optimized geometry. The hydrogen bond between Cys(1)-CO and Cys(4)-NH which has been found in the crystal structure is also found in the optimized geometry. Additionally, the gas phase optimized structure features a hydrogen bond between Cys(1)-CO and Gly-NH.

For the first step of the conformational analysis, the ring conformation was kept essentially unchanged, while the relative conformations of the terminating Boc and OMe ester groups

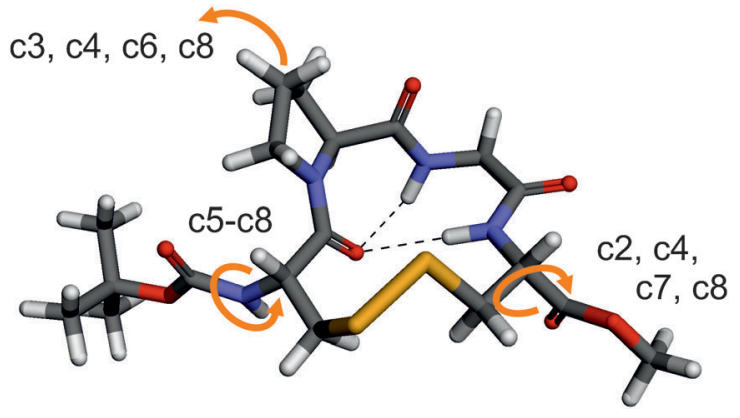

Fig. 3 Optimized geometry of 1 in the gas phase $(r 1-c 1)$. The arrows indicate conformational changes in the side group conformers $c 2$ to $c 8$.

Table $2 \beta$-turn structures in the gas phase optimized ring conformations. Angles are in degrees

\begin{tabular}{llccc}
\hline Structure & $\phi(i+1)$ & $\psi(i+1)$ & $\phi(i+2)$ & $\psi(i+2)$ \\
\hline$\beta_{\mathrm{I}}$ ideal & -60 & -30 & -90 & 0 \\
$\beta_{\text {II }}$ ideal & -60 & 120 & 80 & 0 \\
$\mathbf{1}$ crystal & -62.1 & 135.6 & 60.4 & 29.4 \\
$\beta_{\text {II }}$ r1-c1 $\left(g^{(-)} g^{(-)} t\right)$ & -77.7 & 103.0 & 78.5 & 9.6 \\
$\beta_{\text {II }}$ r7-c1 $\left(g^{(-)} g^{(+)} g^{(+)}\right)$ & -75.6 & 105.4 & 113.1 & -16.8 \\
$\beta_{\text {II }}$ r6-c1 $\left(\operatorname{tg}^{(+)} g^{(+)}\right)$ & -83.3 & 81.3 & 125.9 & -6.7 \\
$\beta_{\mathrm{I}} \mathrm{r} 2-\mathrm{c} 1\left(g^{(-)} g^{(-)} t\right)$ & -85.7 & -6.7 & -116.5 & 26.6 \\
$\beta_{\mathrm{I}} \mathrm{r} 4-\mathrm{c} 1\left(\mathrm{tg}^{(+)} g^{(+)}\right)$ & -83.1 & -3.8 & -107.2 & 10.0
\end{tabular}

as well as the proline ring were modified. This procedure yielded a total of eight different side group conformations (denoted $\mathrm{c} 1$ to $\mathrm{c} 8$, shown in Fig. S3 of the ESI $\dagger$ ). In all but conformers $\mathrm{c} 1$ and $\mathrm{c} 2$, the hydrogen bond between Cys(1)-CO and Gly-NH was found to be cleaved.

Raman measurements of $\mathbf{1}$ and $\mathbf{D}-\mathbf{2}$ indicate that the S-S bridge adopts different conformations ( $c f$. ESI $\dagger$ ). The conformation of disulfide bridges are typically described by the three torsional angles of the $\mathrm{C}^{\alpha}-\mathrm{C}-\mathrm{S}-\mathrm{S}-\mathrm{C}-\mathrm{C}^{\alpha}$ unit as gauche-gauchegauche (ggg), trans-gauche-gauche (tgg or ggt), and trans-gauchetrans $($ tgt). In its crystal structure and in the optimized gas phase structure (Fig. 3), the tetrapeptide 1 adopts a $g^{(-)} g^{(-)} t$ conformation. For 1 with a $\beta_{\text {II }}$ turn structure and the side groups adopting conformation $\mathrm{c} 1$, additional ring conformations with $g^{(+)} g^{(-)} t, g^{(+)} g^{(-)} g^{(+)}, g^{(-)} g^{(+)} g^{(+)}, t^{(+)} g^{(+)}, \operatorname{tg}^{(-)} \mathrm{g}^{(+)}$geometries of the disulfide bridge were generated (Fig. S4, ESI $\dagger$ ).

For two of the SS bridge conformations, namely the $g^{(-)} g^{(-)} t$, and the $\operatorname{tg}^{(+)} g^{(+)}$conformations, the $\beta_{\mathrm{II}}$ has been changed to a $\beta_{\mathrm{I}}$ turn structure (Table 2 and Fig. S5, ESI $\dagger$ ). For both $\beta_{\mathrm{I}}$-tgg conformations, the effects of conformational changes of the side groups have been evaluated as well by generating all eight side group conformations.

After this comprehensive conformational analysis, implicit solvation effects of chloroform and acetonitrile have been taken into account by repeating the geometry optimizations for the $\beta_{\mathrm{II}}$ ring geometries $\mathrm{r} 1$ and $\mathrm{r} 6$ as well as for the corresponding $\beta_{\mathrm{I}}$ structures r2 and r4, applying the integral equation formalism of the polarizable continuum model (IEFPCM) ${ }^{28-30}$ Unlike for the gas phase structures, the hydrogen bond between Cys(1)-CO and Gly-NH was found to be cleaved for the r1-c1 conformer (Fig. S6, ESI $\dagger$ ). 
For all the obtained optimized structures, IR and VCD spectra calculations were carried out.

A conformational analysis of the highly flexible molecule $\mathbf{1}$ based on static structures certainly cannot cover all possible conformations. Furthermore, explicit solute-solvent interactions, especially with the polar solvents, will also influence the relative population of the conformers and completely change energetic preferences. Therefore, the calculated energy differences between the conformers are of low significance, although some trends can be derived. For instance, the sidegroup conformations c5-c8 are typically far less favoured than the first four. All energy differences are given in the ESI $\dagger$ (Table S1). Therefore, instead of the analysis of the VCD spectra of 1 based on energetic differences, we focus on similarities between the experimental and calculated spectral patterns.

Fig. 4 exemplifies the clear trend in the calculated VCD spectral pattern for the $\beta_{\text {II }}$ structures $\mathrm{r} 1-\mathrm{c} 1$ and $\mathrm{r} 6-\mathrm{c} 1$ and their corresponding $\beta_{\mathrm{I}}$ structures $\mathrm{r} 2-\mathrm{c} 1$ and $\mathrm{r} 4-\mathrm{c} 1$. The complete set of calculated VCD spectra is shown in Fig. S7-S9 of the ESI. $\dagger$ In general, the amide I region $\left(1800-1600 \mathrm{~cm}^{-1}\right)$ is found to be much more sensitive to conformational changes of the side group than the amide II region (1600-1480 $\mathrm{cm}^{-1}$ ) which contains mostly contributions of the terminal Boc and methyl ester groups.

Overall, most of the calculated VCD spectra resemble the strong experimental -/+ VCD couplet found in the amide I region reasonably well. However, there are no obvious characteristics in the spectra which could be assigned to either $\beta_{\text {II }}$ or $\beta_{\text {I }}$ turn structures. On the other hand, a significant and more characteristic change is observed for the amide II region when comparing $\beta_{\text {II }}$ and $\beta_{\text {I }}$ structures. The amide II of the $\beta_{\text {II }}$ structures features a $-/+/(+)$ pattern which resembles the experimentally observed amide II region of $\mathbf{1}$ in chloroform and D-2 in all three solvents. In contrast, the amide II pattern is inverted for $\beta_{\mathrm{I}}$ structures showing a $+/-/-$ or $+/-/+$ pattern. This pattern is very similar to the experimental amide II of $\mathbf{L}-\mathbf{2}$ and of $\mathbf{1}$ in polar solvents. This comparison of spectral patterns suggests that D-2 adopts predominantly a $\beta_{\text {II }}$ turn structure, while $\mathbf{L}-2$ preferably resides in a $\beta_{\mathrm{I}}$ turn. For peptide 1, significant contributions of both $\beta_{\mathrm{I}}$ and $\beta_{\mathrm{II}}$

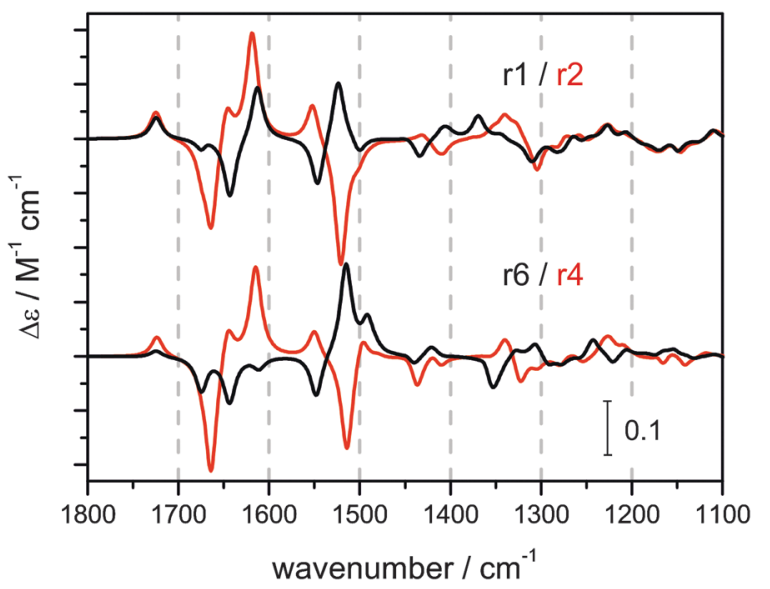

Fig. 4 Calculated VCD spectra of the $\beta_{\|}$structures $r 1-c 1$ and $r 6-c 1$ and the corresponding $\beta_{1}$ structures $\mathrm{r} 2-\mathrm{c} 1$ and $\mathrm{r} 4-\mathrm{c} 1$ (IEFPCM acetonitrile). type structures to the overall conformational equilibrium in polar solvents has to be assumed.

Explicit solute-solvent interactions can affect not only the energetic preferences of certain conformers, but, in general, also influence the calculated IR and VCD intensities. Therefore, we briefly considered explicit solvation for $\mathrm{r} 1-\mathrm{c} 1$ and $\mathrm{r} 2-\mathrm{c} 1$. One acetonitrile molecule was placed in a hydrogen bond with the Gly-NH in $\mathrm{r} 1-\mathrm{c1}$ and r2-c1. This, however, only affected the negative VCD band of the amide II region of the $\beta_{\mathrm{I}}$ structure, so that the spectra obtained look very similar to those without solvent molecules. The corresponding calculated spectra are shown in the ESI $\dagger$ (Fig. S11).

\section{Replica exchange MD of 1}

In our initial study on $\mathbf{1}$, we only carried out short MD calculations (500 ps) without replica exchange. ${ }^{24}$ The study on $\mathbf{D}-2$ and $\mathbf{L}-2$, however, showed that REMD can help to ensure that the calculations do not get trapped in the local energy minimum of the $\beta_{\text {II }}$ turn structure. ${ }^{26}$ Therefore, we carried out additional REMD simulations on $\mathbf{1}$ for the present study. Starting from a crystal structure like $\beta_{\text {II }}$ conformation, the simulations show that the $\beta_{\text {II }}$ motif is basically conserved for $\mathbf{1}$ in acetonitrile, not only at $300 \mathrm{~K}$ but also at higher temperatures (Table 3). A small contribution of $\beta_{\text {I }}$ structures was found representing only $7 \%$ of the total number of structures in the $300 \mathrm{~K}$ simulation and about $14 \%$ in the $400 \mathrm{~K}$ runs. These conformers rapidly converted back to the preferred $\beta_{\text {II }}$ motif. This was corroborated by additional simulations with a $\beta_{\text {I }}$ structure as starting point (Fig. S14, ESI $\dagger$ ). This observation proves that the energy barrier for the conversion from $\beta_{\mathrm{II}}$ to $\beta_{\mathrm{I}}$ is much higher than in the opposite direction. Furthermore, the new REMD simulations show that 1 does indeed adopt the $\beta_{\mathrm{I}}$ turn structure, although only to a very small extent. On the other hand, this small amount of $\beta_{\mathrm{I}}$ structures in 1 may increase with longer simulation times.

\section{Comparison with NMR results and MD data}

For the tetrapeptide $\mathbf{D}-\mathbf{2}$, the VCD analysis points to a predominant $\beta_{\text {II }}$ turn structure. This is consistent with the previous experimental findings from 2D-NMR studies and theoretical REMD simulations. ${ }^{26}$ For the peptide $\mathrm{L}-2$, the interpretation of the VCD spectra suggests a dominant $\beta_{\mathrm{I}}$ turn conformation, while in 2D-NMR the presence of both $\beta_{\mathrm{II}}$ and $\beta_{\mathrm{I}}$ turns was observed, ${ }^{26}$ although it was not possible to use the NMR data to quantify the relative preferences of the turn structures.

For the glycine-containing peptide 1, the previous 2D-NMR of 1 in $\mathrm{CD}_{3} \mathrm{CN}$ showed some clear indications for a $\beta_{\mathrm{II}}$ turn, while the cross-peaks, characteristic for $\beta_{\mathrm{I}}$ turns, were unfortunately

Table 3 Backbone angles $\Phi$ and $\Psi$ calculated for representative structures obtained using cluster analysis at $300 \mathrm{~K}$ and $400 \mathrm{~K}$ and averaged over the trajectory (REMD simulation, $300 \mathrm{~K}$ trajectory). Angles are in degrees and distances in $\AA$

\begin{tabular}{llllll}
\hline & $\phi(i+1)$ & $\psi(i+1)$ & $\phi(i+2)$ & $\psi(i+2)$ & $\mathrm{C}_{\text {Cys } 1}^{\alpha}-\mathrm{C}_{\text {Cys2 }}^{\alpha}$ \\
\hline $300 \mathrm{~K}$ & -79 & 102 & 110 & -16 & 5.77 \\
$400 \mathrm{~K}$ & -75 & 92 & 116 & -9 & 5.63 \\
REMD & $-76 \pm 8$ & $103 \pm 16$ & $106 \pm 10$ & $-11 \pm 15$ & $5.7 \pm 0.2$ \\
& & & & & $<7.0$
\end{tabular}


overlapping with other signals. ${ }^{24}$ Therefore, the presence or absence of $\beta_{\mathrm{I}}$ could not be proven experimentally with NMR spectroscopy.

It is noted that the VCD band of $\mathbf{1}$ in the amide II region is relatively weak in comparison to those of $\mathbf{D}^{-}$and L-2. Furthermore, the VCD spectrum resulting from a simple average of the experimental VCD spectra of $\mathbf{D}^{-}$and $\mathbf{L}-\mathbf{2}$ in either polar solvent is remarkably similar to the spectrum of $\mathbf{1}$ in the same solvent (cf. Fig. S2, ESI $\dagger$ ). These two observations are important since they allow us to conclude that 1 indeed adopts a $\beta_{\mathrm{I}}$ structure to a certain extent in polar solvents although this preference for $\beta_{I}$ is estimated to be only half as much as for $\mathbf{L}-2$. In chloroform, the conformational equilibrium of $\mathbf{1}$ is slightly shifted towards $\beta_{\mathrm{II}}$.

The REMD simulations also qualitatively support these conclusions. The relative trend - $\mathbf{L}-\mathbf{2}$ prefers the $\beta_{\mathrm{I}}$ turn twice as much as $\mathbf{1}$ - is also established.

\section{Conclusions}

In the present work, we apply VCD spectroscopy to study the $\beta$-turn conformations of three cyclic tetrapeptides: ${ }^{24,26} \mathbf{1}, \mathrm{D}-$, and $\mathrm{L}-2$, in the three different solvents, namely chloroform- $\mathrm{d}_{1}$, acetonitrile- $\mathrm{d}_{3}$, and DMSO- $\mathrm{d}_{6}$. The comparison of experimental and theoretical VCD spectra supports the previous finding ${ }^{24,26}$ that $\mathbf{D}-2$ preferably features a $\beta_{\mathrm{II}}$ turn structure. For $\mathbf{L}-2$, the VCD analysis clearly identifies the $\beta_{\mathrm{I}}$ turn as the major conformation. This advances the previous studies where evidence for both $\beta_{\text {II }}$ and $\beta_{\mathrm{I}}$ turn structures was reported although no relative preferences could be quantified. The most important and surprising finding in the present study, however, is that peptide 1 preferably adopts a $\beta_{\mathrm{I}}$ structure in polar solvents, whereas previous NMR evidence for $\beta_{\mathrm{I}}$ was obscured. The current study further demonstrates that this equilibrium between $\beta_{\text {II }}$ and $\beta_{\text {I }}$ turns for 1 depends on the polarity of the solvent. These results therefore reveal a high flexibility of the peptide $\mathbf{1}$.

In summary, this study shows that not only can the chirality of a single amino acid change conformational preferences from $\beta_{\text {II }}$ to $\beta_{\mathrm{I}}$, but also solvent polarity can have significant effects. Furthermore, VCD spectroscopy can provide characteristic spectral patterns of $\beta_{\mathrm{II}}$ and $\beta_{\mathrm{I}}$ turns to complement NMR studies of secondary structural preferences, especially when unresolved or overlapping NMR peaks prevent one from reaching a conclusion.

In future work, we plan to investigate other cyclic tetrapeptides in order to establish a generalized correlation between the VCD spectral pattern and the adopted secondary structure. We will further study the solvent-dependence of the conformational preferences and also the structural changes caused by ring opening reactions.

\section{Acknowledgements}

C.M. acknowledges financial support from the Alexander von Humboldt foundation for a Feodor Lynen Postdoctoral fellowship during his stay at the University of Alberta. E.S.-G. and C.M. thank the Fonds der chemischen Industrie for a Liebig fellowship. This research was supported by the University of Alberta, the Natural Sciences and Engineering Research Council of Canada, and the Canada Research Chairs Program, as well as the Deutsche Forschungsgemeinschaft (DFG) through the Cluster of Excellence RESOLV (EXC 1069). We also gratefully acknowledge access to the computing facilities provided by the Western Canada Research Grid (Westgrid).

\section{Notes and references}

1 C. M. Venkatachalam, Biopolymers, 1968, 6, 1425-1436.

2 P. N. Lewis, F. A. Momany and H. A. Scheraga, Biochim. Biophys. Acta, 1973, 303, 211-229.

3 E. Vass, M. Hollósi, F. Besson and R. Buchet, Chem. Rev., 2003, 103, 1917-1954.

4 L. A. Nafie, Vibrational Optical Actvity, John Wiley \& Sons Ltd, UK, 2011.

5 T. A. Keiderling, in Circular Dichroism. Principles and Applications, ed. K. Nakanishi, N. Berova and R. W. Woody, VCH Publishers Inc., Weinheim and New York and Cambridge, 1994, pp. 497-522.

6 T. A. Keiderling, Curr. Opin. Chem. Biol., 2002, 6, 682-688.

7 E. W. Blanch, L. Hecht and L. D. Barron, Methods, 2003, 29, 196-209.

8 J. Hudecová, J. Horníček, M. Buděšínský, J. Šebestík, M. Šafařík, G. Zhang, T. A. Keiderling and P. Bouř, ChemPhysChem, 2012, 13, 2748-2760.

9 C. Johannessen, R. Pendrill, G. Widmalm, L. Hecht and L. D. Barron, Angew. Chem., Int. Ed., 2011, 50, 1-4.

10 C. Merten, M. Amkreutz and A. Hartwig, Phys. Chem. Chem. Phys., 2010, 12, 11635-11641.

11 C. Merten, L. D. Barron, L. Hecht and C. Johannessen, Angew. Chem., Int. Ed., 2011, 50, 9973-9976.

12 C. Merten, K. Hiller and Y. Xu, Phys. Chem. Chem. Phys., 2012, 14, 12884-12891.

13 J. João Marcos Batista, A. N. L. Batista, D. Rinaldo, W. Vilegas, Q. B. Cass, V. S. Bolzani, M. J. Kato, S. N. López, M. Furlan and L. A. Nafie, Tetrahedron: Asymmetry, 2010, 21, 2402-2407.

14 A.-C. Chamayou, S. Lüdeke, V. Brecht, T. B. Freedman, L. A. Nafie and C. Janiak, Inorg. Chem., 2011, 50, 11363-11374.

15 H. Sato and A. Yamagishi, Int. J. Mol. Sci., 2013, 14, 964-978.

16 V. P. Nicu, E. Debie, W. Herrebout, B. V. der Veken, P. Bultinck and E. J. Baerends, Chirality, 2010, 21, E287-E297.

17 E. D. Gussem, P. Bultinck, M. Feledziak, J. MarchandBrynaert, C. V. Stevens and W. Herrebout, Phys. Chem. Chem. Phys., 2012, 14, 8562-8571.

18 A. Perczel, M. Hollosi, B. M. Foxman and G. D. Fasman, J. Am. Chem. Soc., 1991, 113, 9772-9784.

19 R. W. Woody, Peptides, Polypeptides \& Proteins, Wiley, New York, 1974.

20 R. W. Woody, Biopolymers, 1978, 17, 1451-1467.

21 H. R. Wyssbrod and M. Diem, Biopolymers, 1992, 32, 1237-1242.

22 P. Xie, Q. Zhou and M. Diem, J. Am. Chem. Soc., 1995, 117, 9502-9508.

23 P. Xie, Q. Zhou and M. Diem, Faraday Discuss., 1994, 99, 233-243. 
24 F. Li, K. Bravo-Rodriguez, C. Phillips, R. Seidel, F. Wieberneit, R. Stoll, N. L. Doltsinis, E. Sanchez-Garcia and W. Sander, J. Phys. Chem. B, 2013, 117, 3560-3570.

25 B. N. N. Rao, A. Kumar, H. Balaram, A. Ravi and P. Balaram, J. Am. Chem. Soc., 1983, 105, 7423-7428.

26 F. Li, K. Bravo-Rodriguez, M. Fernandez-Oliva, J. M. RamirezAnguita, K. Merz, M. Winter, C. W. Lehmann, W. Sander and E. Sanchez-Garcia, J. Phys. Chem. B, 2013, 117, 10785-10791.

27 M. J. Frisch, G. W. Trucks, H. B. Schlegel, G. E. Scuseria, M. A. Robb, J. R. Cheeseman, G. Scalmani, V. Barone, B. Mennucci, G. A. Petersson, H. Nakatsuji, M. Caricato, X. Li, H. P. Hratchian, A. F. Izmaylov, J. Bloino, G. Zheng, J. L. Sonnenberg, M. Hada, M. Ehara, K. Toyota, R. Fukuda, J. Hasegawa, M. Ishida, T. Nakajima, Y. Honda, O. Kitao, H. Nakai, T. Vreven, J. J. A. Montgomery, J. E. Peralta, F. Ogliaro, M. Bearpark, J. J. Heyd, E. Brothers, K. N. Kudin, V. N. Staroverov, T. Keith, R. Kobayashi, J. Normand, K. Raghavachari, A. Rendell, J. C. Burant, S. S. Iyengar, J. Tomasi, M. Cossi, N. Rega, J. M. Millam, M. Klene, J. E. Knox, J. B. Cross, V. Bakken, C. Adamo, J. Jaramillo, R. Gomperts, R. E. Stratmann, O. Yazyev, A. J. Austin, R. Cammi, C. Pomelli, J. W. Ochterski, R. L. Martin, K. Morokuma, V. G. Zakrzewski, G. A. Voth, P. Salvador, J. J. Dannenberg, S. Dapprich, A. D. Daniels, O. Farkas, J. B. Foresman, J. V. Ortiz, J. Cioslowski and D. J. Fox, Gaussian 09, Revision C.01, Gaussian, Inc., Wallingford CT, 2010.

28 B. Mennucci, C. Cappelli, R. Cammi and J. Tomasi, Chirality, 2011, 23, 717-729.
29 B. Mennucci, J. Tomasi, R. Cammi, J. R. Cheeseman, M. J. Frisch, F. J. Devlin, S. Gabriel and P. J. Stephens, J. Phys. Chem. A, 2002, 106, 6102-6113.

30 J. Tomasi, B. Mennucci and R. Cammi, Chem. Rev., 2005, 105, 2999-3094.

31 B. Hess, C. Kutzner, D. van der Spoel and E. Lindahl, J. Chem. Theory Comput., 2008, 4, 435-447.

32 T. Okabe, M. Kawata, Y. Okamoto and M. Mikami, Chem. Phys. Lett., 2001, 335, 435-439.

33 E. Guàrdia, R. Pinzón, J. Casulleras, M. Orozco and F. J. Luque, Mol. Simul., 2001, 26, 287-306.

34 G. A. Kaminski, R. A. Friesner, J. Tirado-Rives and W. L. Jorgensen, J. Phys. Chem. B, 2001, 105, 6474-6487.

35 A. Patriksson and D. van der Spoel, Phys. Chem. Chem. Phys., 2008, 10, 2073-2077.

36 U. Essmann, L. Perera, M. L. Berkowitz, T. Darden, H. Lee and L. G. Pedersen, J. Chem. Phys., 1995, 103, 8577-8593.

37 M. Tuckerman, B. J. Berne and G. J. Martyna, J. Chem. Phys., 1992, 97, 1990-2001.

38 B. Hess, H. Bekker, H. J. C. Berendsen and J. G. E. M. Fraaije, J. Comput. Chem., 1997, 18, 1463-1472.

39 X. Daura, K. Gademann, B. Jaun, D. Seebach, W. F. van Gunsteren and A. E. Mark, Angew. Chem., Int. Ed., 1999, 38, 236-240.

40 J. R. Cheeseman, M. J. Frisch, F. J. Devlin and P. J. Stephens, Chem. Phys. Lett., 1996, 252, 211-220.

41 P. J. Stephens, J. Phys. Chem., 1985, 89, 748-752.

42 F. J. Devlin, P. J. Stephens, J. R. Cheeseman and M. J. Frisch, J. Phys. Chem. A, 1997, 101, 6322-6333. 